\title{
Energy Efficient WSN-SAT System for Surveillance of Satellites
}

\author{
Hafiz Bilal Khalil, Naeem Abas, Shoaib Rauf \\ Department of Electrical Engineering, University of Gujrat, Gujrat, Pakistan \\ Email: Engr.bilalkhalil@yahoo.com, naeemkalair@uog.edu.pk, engr.shoaib@uog.edu.pk
}

Received 17 June 2015; accepted 9 October 2015; published 12 October 2015

Copyright (C) 2015 by authors and Scientific Research Publishing Inc.

This work is licensed under the Creative Commons Attribution International License (CC BY). http://creativecommons.org/licenses/by/4.0/

c) (i) Open Access

\begin{abstract}
Satellite communication plays a vital role in the arena of telecommunication. The main objective of this manuscript is to examine the threats and problems like space debris, space weather and space weapons that could damage or disable the satellite system. In consequence, we discuss the role of wireless sensor networks (WSN) and nodes in space based technologies. The scope of this paper is to minimize the threats and vulnerabilities to the satellite systems and based on that, design the next generation and state of the art satellite communication system. We proposed the solutions for the vulnerabilities and surveillance of satellite system by integrating the energy efficient and cost effective WSN to the satellite system.
\end{abstract}

\section{Keywords}

Energy Efficiency, Satellite Communication, Wireless Sensor Networks, Space Threats, Space Debris

\section{Introduction}

Satellite communication system enormously developed in the first decade of the 21st century. At the same time with the pace of technology augmentation, satellite communication has developed its applications for military operations, education, business, transportation tracking, navigation, television broadcasting, teleconferences, weather forecasting, health and many more. Most of the developed countries are now satellite dependent in order to conduct their routine work. The satellite system becomes more vulnerable to failure due to various factors, such as the space debris, space weather and anti-satellite missiles [1] [2]. In 2010 the International Telecommunication Union (ITU) in its recommendation S.1003-2 has declared that there are 1100 known spacecraft and rocket bodies near the Geosynchronous orbit (GSO) and one-third of them are currently active [3]. Due to the Earth-based observations, we have limited information about the geostationary orbit (GEO) environment. In low 
Earth orbit (LEO) we can detect the smallest object having $30 \mathrm{~cm}$ dimensions while in best conditions the smallest object that can be detected in GEO is almost $1 \mathrm{~m}$ in dimensions [4]. This debris can produce more debris in collision with the inactive or lost satellite while it is also the risk for the active satellites. To reduce the collisions with debris we must have more knowledge about the geostationary environment. We can send a satellite for the observation, but after that mission it will also cause to create more fragmentations [5].

In addition, space weather is another problem faced by the satellite system as the space weather is unpredictable and more dynamic. Space weather is the byproduct of solar activity and has high energy electrons that affect the satellite. The surface of the Sun radiates the high energy electron during the solar activity. These electrons travel towards the Earth and also cause vulnerabilities in GEO system. However, these satellites are shielded for protection, but with the passage of time this shielding becomes weakened and degraded. When a satellite is damaged it is very difficult to get it back and analyze the reasons behind the failure. It becomes necessary to monitor the effects on the surface of the satellite at the end of its operational life. So, sending another satellite to monitor the effects is not economical and wise. We need an alternate system that should be low cost, energy efficient, flexible and small in size to sense and communicate the required information without creating more debris.

After extensive research, we found all these characteristics in the wireless sensor networks (WSN). We proposed integrated wireless sensor-satellite system (I-WSAT) system to approach the goals. Then WSN is widely used in monitoring, military operations, inventory control, health, tracking, sensing of vibration, intuiting heat and various atmospheric parameters not only on earth but also under the sea [6]. However, these wireless sensor nodes cannot be implemented in the spacecraft surface and orbit [7]. Special purpose, optimized and modified nodes will be required to communicate the sensed information in the space missions. Besides this, for WSN nodes a radio communication link should be designed to directly communicate with satellite [8].

The objective of this paper is to monitor the space threats without sending satellites into the space. We suggest the Wireless sensor networks to monitor the orbits and outer surface of the satellites. In the following sections, this paper will describe the threats to the satellite system and need of WSN. Furthermore, we explain the challenges and opportunities in the implementation of WSN node in space and orbits.

\section{Wireless Sensor Networks (WSN)}

Wireless sensor network is the collection of small nodes that sense the information and transmit data to the main node. These micro sensors consists sensing module, radio module, digital signal processing unit and an energy source. Wireless sensor network works cooperatively they sends their own data and also act as router to send the sensed information of other nodes. Each node sends the information of other nodes towards the main node according to predefined routing protocols. Applications of WSN are exponentially expanded in monitoring, surveillance, intelligence, protection and control of the environment or hard to go areas. Figure 1 describes the role

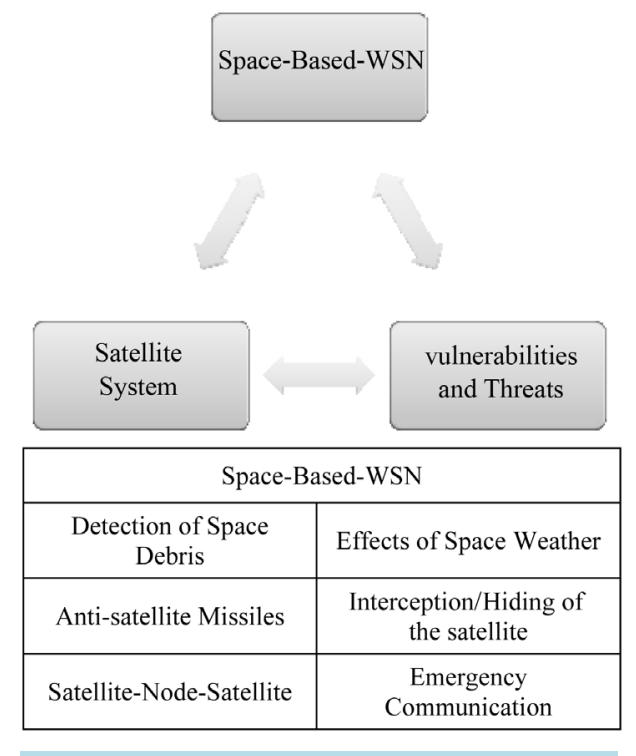

Figure 1. Role of WSN in satellite system. 
of WSN for detection of various vulnerabilities like detection of debris, effects of space weather and emergency communication etc. in satellite system.

\section{Vulnerabilities and Threats to Satellite System}

\subsection{Protection of GSO}

There are some limitations to retrieve objects to the Earth from geostationary orbit (GSO) at the end of their operational life. In [3] ITU has also recommended that a region must be established above or below the GSO where the operational satellite may reside and maneuver. And at the end of operational life the satellites should be taken out of this region. Spacecraft operator should assure the availability of fuel to achieve the required maneuver at the end of satellite life. This dedicated region for active satellite should be free from debris and any other inactive satellite to reduce the risk of collision. To monitor the dedicated region, we cannot put additional satellite because it can cause some additional debris after its operational life. In this scenario WSN is the best options for the monitoring of debris of GSO.

\subsection{Space Weather}

Space weather has effects on the surface of the satellites and space weather is more dynamic than the predicted models. The space weather mostly affected the Geo stationary satellites [2]. So, Geo satellites are designed by taking the space weather into the account, but it is not enough. We need more information regarding this issue, so we need to study the inactive and failure satellites caused by the space weather. It is very difficult to bring back the satellite to study the weather effects on it. To study the space weather effects on the satellite WSN can play a vital role in continuous monitoring of the surface of satellite, and we can design more secure and space weather resistant satellites in the future. It can improve the life and efficiency of the GEO satellite and provide help in preparing the next generation communication satellites.

\subsection{Space Weapon}

Space weapons are the biggest threat to the satellite system. Anti-satellite weapons are capable to take the warhead into the space. Many countries such as Pakistan, China, Russia, Iran and India have made such ballistic missiles that can take the low-yield nuclear weapon into the space. Explosion of such type weapon in LEO can destroy the satellite [9] and shorten the life cycle of the satellite. China has already successfully conducted antisatellite weapon (ASAT) experiment to destroy its weather satellite, which is alarming. The Low-altitude-shortduration-orbital ASAT and High-altitude-short-duration-orbital ASAT are interceptors that are launched from the ground into a temporary parking orbit from which they maneuvers to attack their respective targeted satellite. The on-orbit lifespan of temporarily parked interceptor is measured in hours; detection of this system is slightly more complex than direct ascent weapons. An operational satellite can be made hide from interceptors or radars using the WSN special nodes, which cover the original signal by transmitting the number of signals at the same frequency [10]. WSN can perform continues monitoring in their targeted region and can detect temporarily parked interceptor.WSN can be used to examine the nuclear effects in LEO and collect the information.

Space to space missile is another threat for the satellite security. Space to space missile system consists of a carrier satellite, missile and control system. A missile can be launched from space to hit the target in the space or another missile targeting the carrier satellite that is coming from the earth or other satellite [11]. WSN covers the larger area in space and gather the information about the other missile carrier satellites and give instructions for precautionary measures in emergency conditions.

Micro-satellite and Nano-satellite perform the satellite inspection and also use in imaging processes [11]. They also can be used as weapons to destroy the target satellite. It can be placed on an intercept course and programmed to hone in satellite or destroy the target satellite. Defense against such type attack would be difficult. But now WSN provides the solution, Nodes can detect any moving object along the satellite. The interceptor satellite can be detected before the explosion and warning can be submitted to the ground station with plenty of time. A precautionary measure can be taken to save that satellite from such type attacks.WSN can be used as parasitic communication, but according to the rules ITU-R S.524-8 and ITU-R SF.358-5 [3] interference should not take place among the other satellites. Nano and Pico satellite concept has already been introduced to interconnect the satellites and for the inspection of satellite [12]. WSN keeps monitoring the surroundings of satellite 
just like Pico-Satellite. WSN can be connected with active and inactive satellites to perform various activities. The synopsis of interconnected I-WSAT has been shown in Figure 2.

\section{Challenges in Implementation of WSN in Space}

\subsection{Deployment}

The deployment of WSN in space can be random or installed at premeditated chosen locations on the outer surface of the satellite or in the surroundings [13]. In physical environment the spreading of WSN nodes can be a continuous process, and nodes can be added time to time according to the requirement and nature of sensing. However, deployment of the nodes is a one-time activity and deployed on satellite or in space at predetermined locations and at the same time in the orbit. Because in case of satellite the replacing of nodes to improve the network and sensing efficiency is not an easy task. WSN nodes can be spread in kilometers around the satellite to detect the debris and cover or hide the satellite from the interceptors. At the initial stage of the space mission the WSN nodes can also be attached with the surface of satellite (as shown in Figure 3) to monitor the various parameters. The deployment cost, mass and classification of previous satellite mission is shown in Table 1.

\subsection{Networking}

In space there will be no infrastructure for networking. WSN nodes can make the ad-hoc network to communicate with each other and communicate with the ground station through the satellite. Each sensed information regarding to the space weather, change in the surface of the satellite, debris collision or any other valuable information should be communicated to the control system of satellite and also to the ground station through the satellite.

Ad hoc networking is the most suitable and preferred networking technique for the WSN nodes as the installation of network infrastructure is costly and not feasible. The combination of adhoc network and infrastructure based (satellite communication) [13] network is the way to communicate the sensed information between the sensor nodes and ground control station. Each sensor transmits the information to all the nodes, while one sink node or gateway sends the information to the satellite. Selection of the node that transmits the information to the satellite is important to increase the performance of the network; MNMU-RA [14] routing algorithm can be chosen to select the main node in WSN.

There are various technologies being used in WSN for networking such as ZigBee (IEEE 802.15.4 Standard), Wi-Fi (IEEE 802.11) and Bluetooth (IEEE 802.15.1) for low data rate and short distance communication. Some recent research has investigated the potential of ultra wideband (UWB) technology for WSN [15]. As we

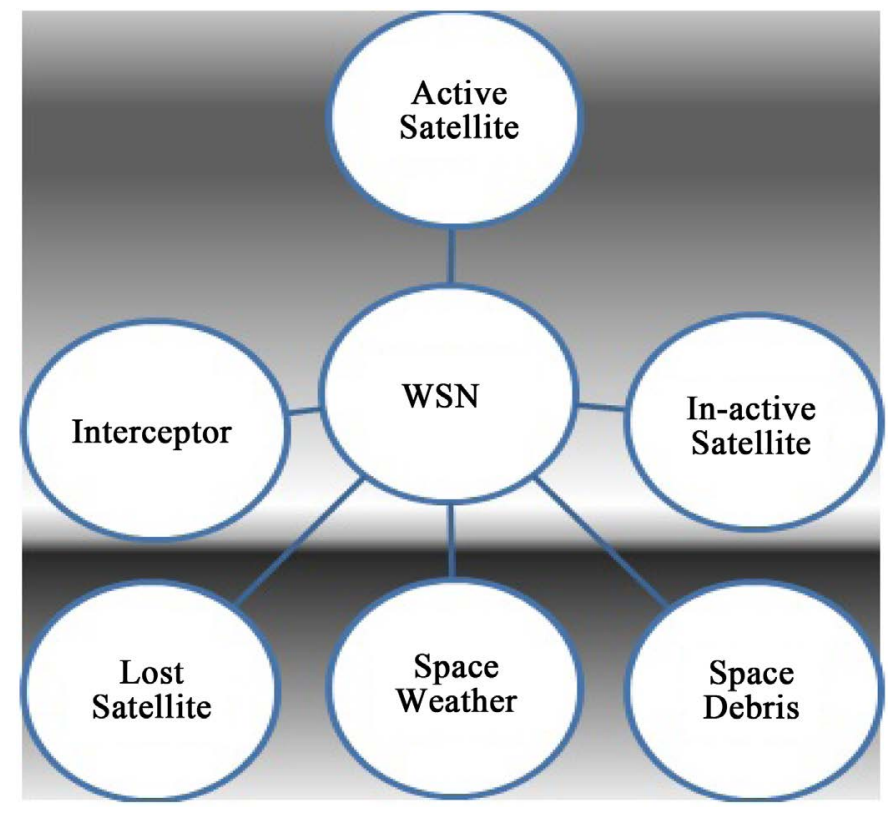

Figure 2. Synopsis of I-WSAT system. 


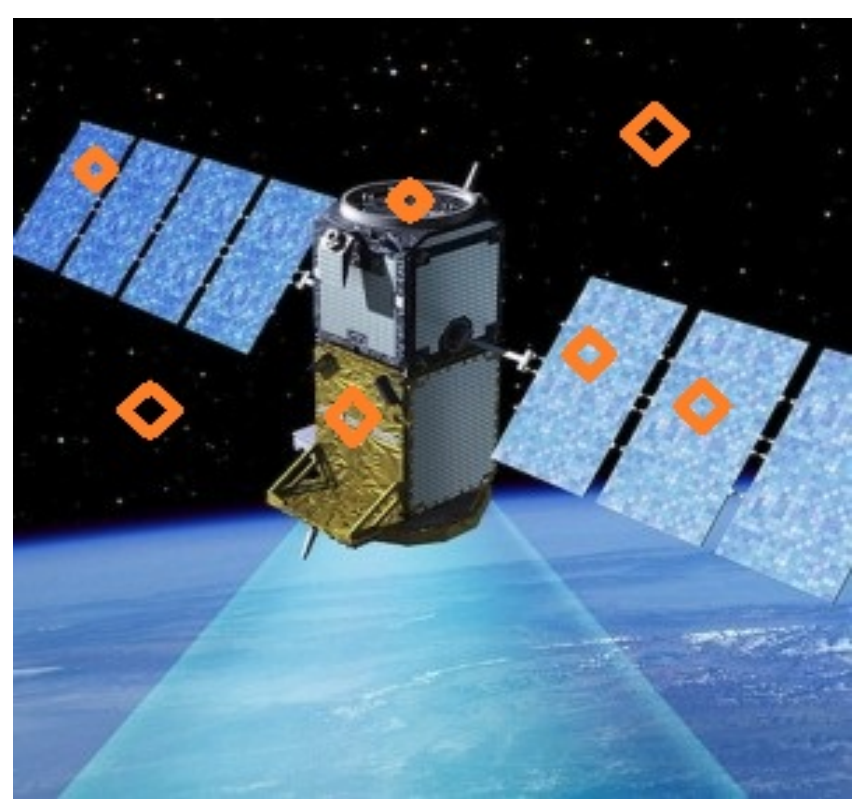

Figure 3. Deployment of WSN on surface of satellite.

Table 1. Classification of satellites.

\begin{tabular}{cccc}
\hline Names of satellite & Mass & Mission Cost & Classification \\
\hline Inmar Sat-4 & $5945 \mathrm{Kg}$ & $\$ 0.1-2 \mathrm{~B}$ & Large \\
GIOVE-A & $660 \mathrm{Kg}$ & $\$ 50-100 \mathrm{M}$ & Medium \\
UK-DMC & $166 \mathrm{Kg}$ & $\$ 10-50 \mathrm{M}$ & Mini \\
PicoSat & $67 \mathrm{Kg}$ & $\$ 2-10 \mathrm{M}$ & Micro \\
SNAP & $6.5 \mathrm{Kg}$ & $\$ 0.2-2 \mathrm{M}$ & Nano \\
PalmSat & $1 \mathrm{Kg}$ & $\$ 20-200 \mathrm{~K}$ & Pico \\
PCB Sat & $250 \mathrm{~g}$ & $\$ 3-20 \mathrm{~K}$ & Femto \\
\hline
\end{tabular}

concerned about the energy efficient WSN, the UWB has the characteristics of energy efficiency. We conduct the simulated experiment in a network and calculate the utilization of energy separately at various standards (Wi-Fi, Bluetooth and UWB) and other parameters are mentioned in Table 2. For simulation 20 nodes are randomly deployed ranging from 1 to 10 meters distance and moving with constant speed around an object (satellite). Same network and topology taken at each time and calculate the results. A comparison of energy consumption in different modes; sensing, receiving and transmission is shown in Figure 5. As the energy consumption is high during the transmission mode, but the results have shown the significant reduction in energy consumption during the transmission mode of WSN nodes.

We proposed satellite-node-satellite communication way to communicate (Figure 4). In this method two satellites can communicate with each other with the help of common connected WSN node. An active satellite can find or detect the inactive satellite with the help of nearby WSN nodes. An efficient link budget is required for the radio signal where WSN can directly communicate with the satellite [16]. While already satellite-satellite communication takes place in between the satellites. This technique will help to locate the lost or completely failed satellite in space with the help of near satellite and WSN nodes in that orbit.

\subsection{Mobility}

Space weather can also have an impact on WSN nodes and can cause in change of their location or even a failure of any single node. It is also a challenge for WSN in space, so the WSN nodes should have the capability to 


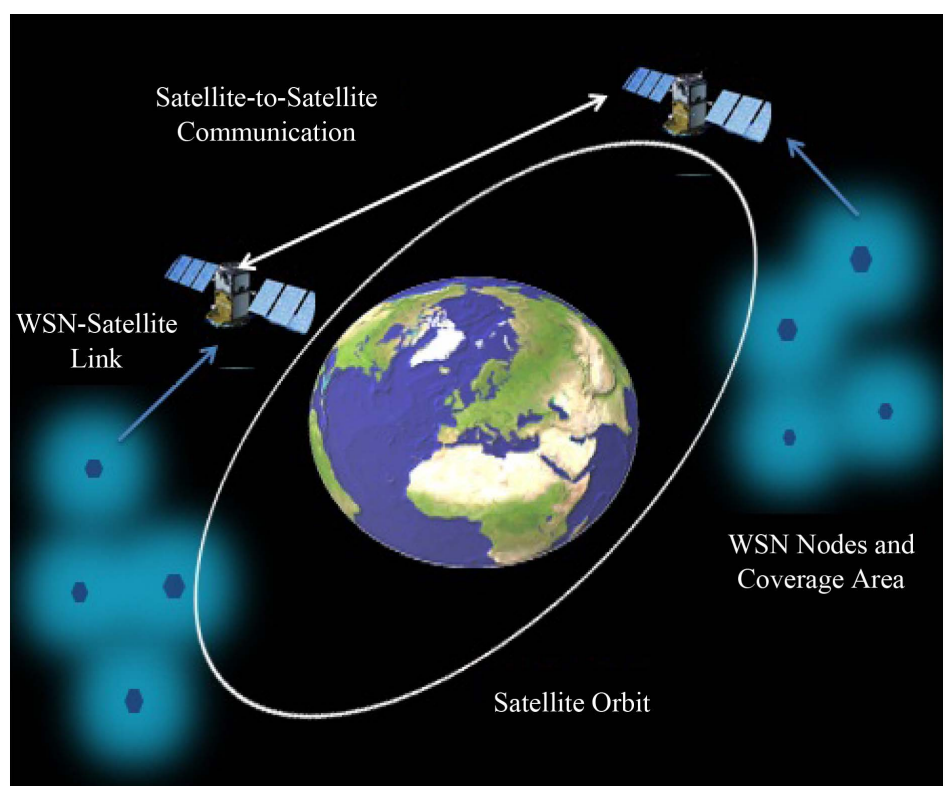

Figure 4. Satellite-WSN-Satellite communication.

Table 2. Parameters for simulation.

\begin{tabular}{|c|c|}
\hline Features/operation & Unit/Detail \\
\hline Sleep & $19 \mu \mathrm{A}$ \\
\hline Receiving & $10 \mathrm{Ma}$ \\
\hline Transmission & $17 \mathrm{Ma}$ \\
\hline Radio Frequency & $2.4 \mathrm{GHz} / 900 \mathrm{MHz} / 500 \mathrm{MHz}$ \\
\hline Bit rate & $420 \mathrm{Kbps}$ \\
\hline Bandwidth & 25 MBPS/ \\
\hline Data packet & 8 Bytes \\
\hline Number of Nodes & 20 \\
\hline Minimum Distance & $\sim 1$ meter \\
\hline Maximum Distance & 10 meters \\
\hline Nodes to Main Node links & 4 \\
\hline Main Nodes to satellite link & 1 \\
\hline
\end{tabular}

maintain the network and compute the new routing path in case of displacement or failure of any node. Networking protocol and algorithm should be designed to take into account that the orbital mobility can have a larger impact on the sensor nodes. For effective surveillance modified and efficient nodes are required, which may have a transmission range in kilometers and flexible to maintain the network and large coverage area. Space environment issues must be considered like shock, vibration and acceleration. Some other atmospheric such as corrosion and vacuum may also have effects on the network. The mobility of WSN nodes is uncontrolled and passive; the speed of movement of nodes should be compatible with the speed of under observation satellite.

\subsection{Energy}

The satellite system has used several integrated micro power systems for electrical energy such as solar cells, fuel cell, nuclear battery, chemical battery, induction and vibration. Energy is the tradeoff between size and cost/ network life [17]. Due to the smaller size, the limited energy source is also a big issue for WSN nodes. Energy is 
stored in small batteries but it can also be harvested from the environment for longer networking life. For space mission, we cannot depend only on batteries or fuel, WSN nodes should be embedded with the small solar panel just like the satellite system. Typical solar cells are fabricated with optimized silicon or gallium arsenide, for efficient power generation the integrated solar cell should be fabricated with CMOS but it is not yet practically implemented [18].

Energy conservation is directly proportional to the life of network. Most of the energy is consumed during the transmission of information, while sensing required minimum amount of energy during the idle mode. Routing algorithms play an important role in energy conservation, for the longer network life an energy efficient routing algorithm must be selected.

After the extensive research, we conclude that efficient utilization of energy, cost effectiveness and smaller size are the key elements for the future satellite missions. A comparison of energy utilization (at different stages) between UWB and our proposed solution has been shown in Figure 5. Simulated results have shown remarkable achievements in energy efficiency. Our proposed Table 3 has shown the some other required features for the WSN-Satellite missions.

\section{Conclusion}

This manuscript describes both the wireless sensor networks and satellite systems. An integrated WSN-Satellite system was proposed with the aim to reduce the vulnerabilities and threats associated with the satellite based technologies. WSN can be a cost effective solution for many problems, but it is not an easy task to use WSN in the space. We describe the challenges in the integration of WSN with the satellite system and proposed various

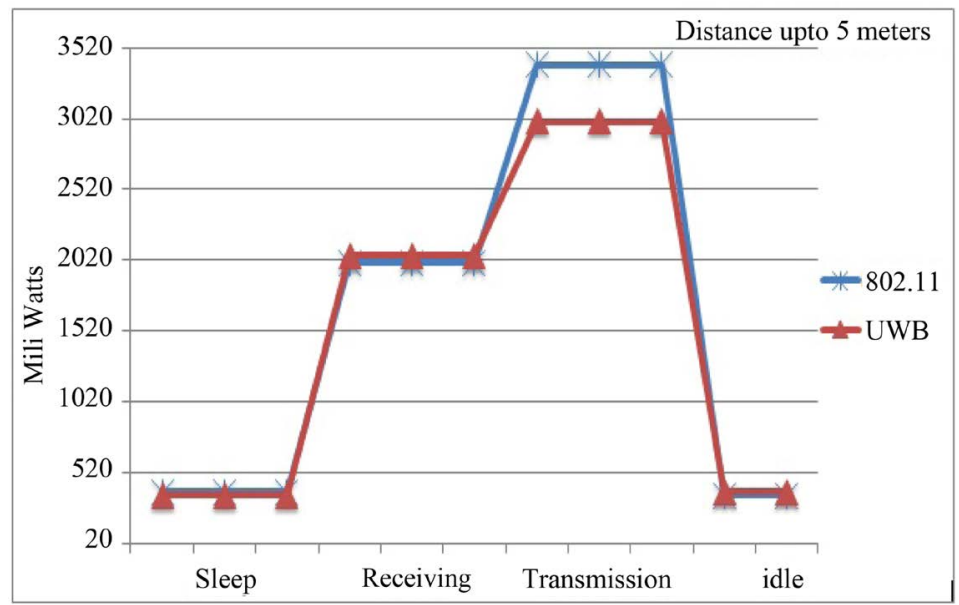

Figure 5. Comparison of power consumption at different operation.

Table 3. Requirement for space WSN nodes.

\begin{tabular}{cc}
\hline WSN Features & Requirement for Space \\
Size & As low as Possible to reduce debris \\
Pass & Minimum \\
Communication & Maximum life cycle required \\
Number of Nodes & Node-to-node, Node to satellite, satellite-Node-satellite. \\
Altitude & $<100$ \\
Coverage & Appropriate for (LEO, GSO or mission) \\
Routing & Satellite surface and surroundings \\
Resilience & Co-operative, \\
\end{tabular}


modifications required in traditional WSN. An advantage of WSN over the parasitic and monitoring satellite is that typical satellite needs explosion or deterioration after the life spans, which increase the fragmentation debris and can be harmful for other satellites in the same orbit. WSN requires no such type explosion. ASAT weapons and interceptors are major threats for the developed and satellite dependent countries; WSN is an effective system to tackle such type of problems. In future, after the required modification and hardware optimization in the nodes WSN-Satellite system would be economical, effective and debris free system for the space missions and surveillance.

\section{References}

[1] Huntington, J. (2007) Improving Satellite Protection with Nanotechnology. Air WarColl Maxwell AFB AL Center for Strategy and Technology, United States Air Force (USAF) Proof. http://oai.dtic.mil/oai/oai?verb=getRecord\&metadataPrefix=html\&identifier=ADA474825

[2] Iucci, N., et al. (2005) Space Weather Conditions and Spacecraft Anomalies in Different Orbits. Space Weather 3.1.

[3] Recommendation ITU-R S.1003-2 (12/2010) Environmental Protection of Geo-Stationary-Satellite Orbit. http://www.itu.int/rec/R-REC-S.1003/_page.print

[4] Schildknecht, T., et al. (2004) Optical Observations of Space Debris in GEO and in Highly-Eccentric Orbits. Advances in Space Research, 34, 901-911. http://dx.doi.org/10.1016/j.asr.2003.01.009

[5] Kitazawa, Y. (2012) Effective Detection of Low-Luminosity GEO Objects Using Population and Motion Predictions. Defense Technical Information Center, Fort Belvoir, VA.

[6] Celandroni, N., et al. (2013) A Survey of Architectures and Scenarios in Satellite-Based Wireless Sensor Networks: System Design Aspects. International Journal of Satellite Communications and Networking, 31, 1-38. http://dx.doi.org/10.1002/sat.1019

[7] Akbulut, A., et al. (2011) Wireless Sensor Networks for Space and Solar-System Missions. 5th International Conference on Recent Advances in Space Technologies (RAST), Istanbul, 9-11 June 2011, 616-618. http://dx.doi.org/10.1109/RAST.2011.5966912

[8] Poulakis, M.I., Vassaki, S. and Panagopoulos, A.D. (2013) Satellite-Based Wireless Sensor Networks: Radio Communication Link Design. 7th European Conference on Antennas and Propagation (EuCAP), Gothenburg, 8-12 April 2013, 2620-2624.

[9] Hagt, E. (2007) China's ASAT Test: Strategic Response. China Security, 3, 31-51.

[10] Kim, K., Lee, M.W. and Lim, J.S. (2012) Spreading Technique of Satellite Beacon to Avoid Jamming Attacks. 14th International Conference on Advanced Communication Technology (ICACT), PyeongChang, 19-22 February 2012, 778-781.

[11] Wilson, T. (2001) Threats to United States Space Capabilities. Prepared for the Commission to Assess United States National Security Space Management and Organization.

[12] Funase, R., et al. (2007) Technology Demonstration on University of Tokyo’s Pico-Satellite “XI-V” and Its Effective Operation Result Using Ground Station Network. Acta Astronautica, 61, 707-711. http://dx.doi.org/10.1016/j.actaastro.2006.12.032

[13] Romer, K. and Mattern, F. (2004) The Design Space of Wireless Sensor Networks. IEEE Wireless Communications, 11, 54-61. http://dx.doi.org/10.1109/MWC.2004.1368897

[14] Khalil, H. and Zaidi, S. (2012) MNMU-RA: Most Nearest Most Used Routing Algorithm for Greening the Wireless Sensor Networks. Wireless Sensor Network, 4, 162-166. http://dx.doi.org/10.4236/wsn.2012.46023

[15] Mahfuz, M.U. and Ahmed, K.M. (2005) A Review of Micro-Nano-Scale Wireless Sensor Networks for Environmental Protection: Prospects and Challenges. Science and Technology of Advanced Materials, 6, 302-306. http://dx.doi.org/10.1016/j.stam.2005.02.008

[16] Akyildiz, I.F., Su, W., Sankarasubramaniam, Y. and Cayirci, E. (2002) Wireless Sensor Networks: A Survey. Computer Networks, 38, 393-422. http://dx.doi.org/10.1016/S1389-1286(01)00302-4

[17] Barnhart, D.J. (2008) Very Small Satellite Design for Space Sensor Networks. Surrey Univ Guildford (United Kingdom) Surrey Space Centre, Guildford.

[18] Edmonson, W., Edmonson, W., Neogi, N. and Herencia-Zapana, H. (2014) Small Satellite Systems Design Methodology: A Formal and Agile Design Process. 8th Annual IEEE Systems Conference (SysCon), Ottawa, 31 March-3 April 2014, 518-524. http://dx.doi.org/10.1109/syscon.2014.6819305 